\title{
A Cryogenic Axial-Centrifugal Compressor For Superfluid Helium Refrigeration
}

L. Decker, K. Löhlein, P. Schustr*, M. Vin_* , I. Brunovsky?, L. Tucek?, Ph. Lebrun? and L. Tavian?

\begin{abstract}
CERN's new project, the Large Hadron Collider (LHC), will use superfluid helium as coolant for its high-field superconducting magnets and therefore require large capacity refrigeration at $1.8 \mathrm{~K}$. This may only be achieved by subatmospheric compression of gaseous helium at cryogenic temperature. To stimulate development of this technology, CERN has procured from industry prototype Cold Compressor Units (CCU). This unit is based on a cryogenic axial-centrifugal compressor, running on ceramic ball bearings and driven by a variable-frequency electrical motor operating under low-pressure helium at ambient temperature. The machine has been commissioned and is now in operation. After describing basic constructional features of the compressor, we report on measured performance.
\end{abstract}

Linde Kryotechnik, CH-8422 Pfungen, Switzerland

* ATEKO,Resslova 13, CS-50010 Hradec Králové, Czech Republic

?PBS, CS-59512 Velká Bite_Czech Republic

? LHC Division, CERN, CH-1211 Geneva 23, Switzerland

ICEC 16/ICMC, Kitakyushu, Japan, 20-24 May 1996

Administrative Secretariat

LHC Division

CERN

CH -1211 Geneva 23

Switzerland

Geneva, 12 July, 1996 


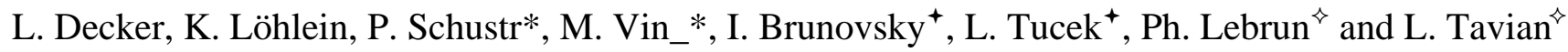

Linde Kryotechnik, CH-8422 Pfungen, Switzerland

* ATEKO,Resslova 13, CS-50010 Hradec Králové, Czech Republic

+ PBS, CS-59512 Velká Bite_, Czech Republic

$\diamond$ LHC Division, CERN, CH-1211 Geneva 23, Switzerland

CERN's new project, the Large Hadron Collider (LHC), will use superfluid helium as coolant for its high-field superconducting magnets and therefore require large capacity refrigeration at $1.8 \mathrm{~K}$. This may only be achieved by subatmospheric compression of gaseous helium at cryogenic temperature. To stimulate development of this technology, CERN has procured from industry prototype Cold Compressor Units (CCU). This unit is based on a cryogenic axialcentrifugal compressor, running on ceramic ball bearings and driven by a variable-frequency electrical motor operating under low-pressure helium at ambient temperature. The machine has been commissioned and is now in operation. After describing basic constructional features of the compressor, we report on measured performance.

\section{INTRODUCTION}

CERN's new project, the Large Hadron Collider (LHC) [1] now under construction, will use superfluid helium as coolant for its high-field superconducting magnets and therefore require large capacity refrigeration at $1.8 \mathrm{~K}$. This may only be achieved by subatmospheric compression of gaseous helium at cryogenic temperature. To stimulate development of this technology, CERN has procured from Linde, with ATEKO and PBS as subcontractors, a prototype Cold Compressor Unit (CCU) with a nominal flowrate of $18 \mathrm{~g} / \mathrm{s} @ 1 \mathrm{kPa}$ inlet pressure, with a pressure ratio of 3 and with an isentropic efficiency better than $60 \%$. These specifications also meet the need for a booster stage enabling to triple the capacity of an existing warm pumping unit (WPU) which provides $1.8 \mathrm{~K}$ refrigeration to the CERN cryogenic test station, an upgrade described in a companion paper [2]. The compliance of the system allows to handle low flow continuously varying over the range from 6 to $18 \mathrm{~g} / \mathrm{s}$, with a pressure ratio of 1 to 3 and with an isentropic efficiency better than 0.5 . For a given mass-flow, the gas inlet temperature may vary depending upon the number of test stations in operation. Table 1 summarizes the main specifications of the CCU.

Table 1 Main specifications of CCU

\begin{tabular}{lcc}
\hline Capacity & Nominal & Low-flow \\
\hline Helium flow-rate [g/s] & 18 & 6 to 18 \\
Suction Pressure [kPa] & 1 & $\leq 1$ \\
Discharge pressure [kPa] & 3 & 1 to 3 \\
Pressure ratio & 3 & - \\
Helium suction temperature [K] & 3.5 to 4.4 & 5.3 to 3.5 \\
Isentropic efficiency & $\geq 0.6$ & $\geq 0.5$ \\
\hline
\end{tabular}

\section{BASIC CONSTRUCTIONAL FEATURES}

General Layout

The main constituent of the CCU is a cold box which houses all components at low temperature. On the top flange, the cold compressor is mounted with vertical axis. A cartridge design allows to remove the rotating part of the compressor without breaking the vacuum of the CCU enclosure. Two bayonet connections to the compressor inlet and outlet constitute the interface with a separate valve box which contains two insulation valves and a bypass valve. Figure 1 shows the general layout of the CCU. 


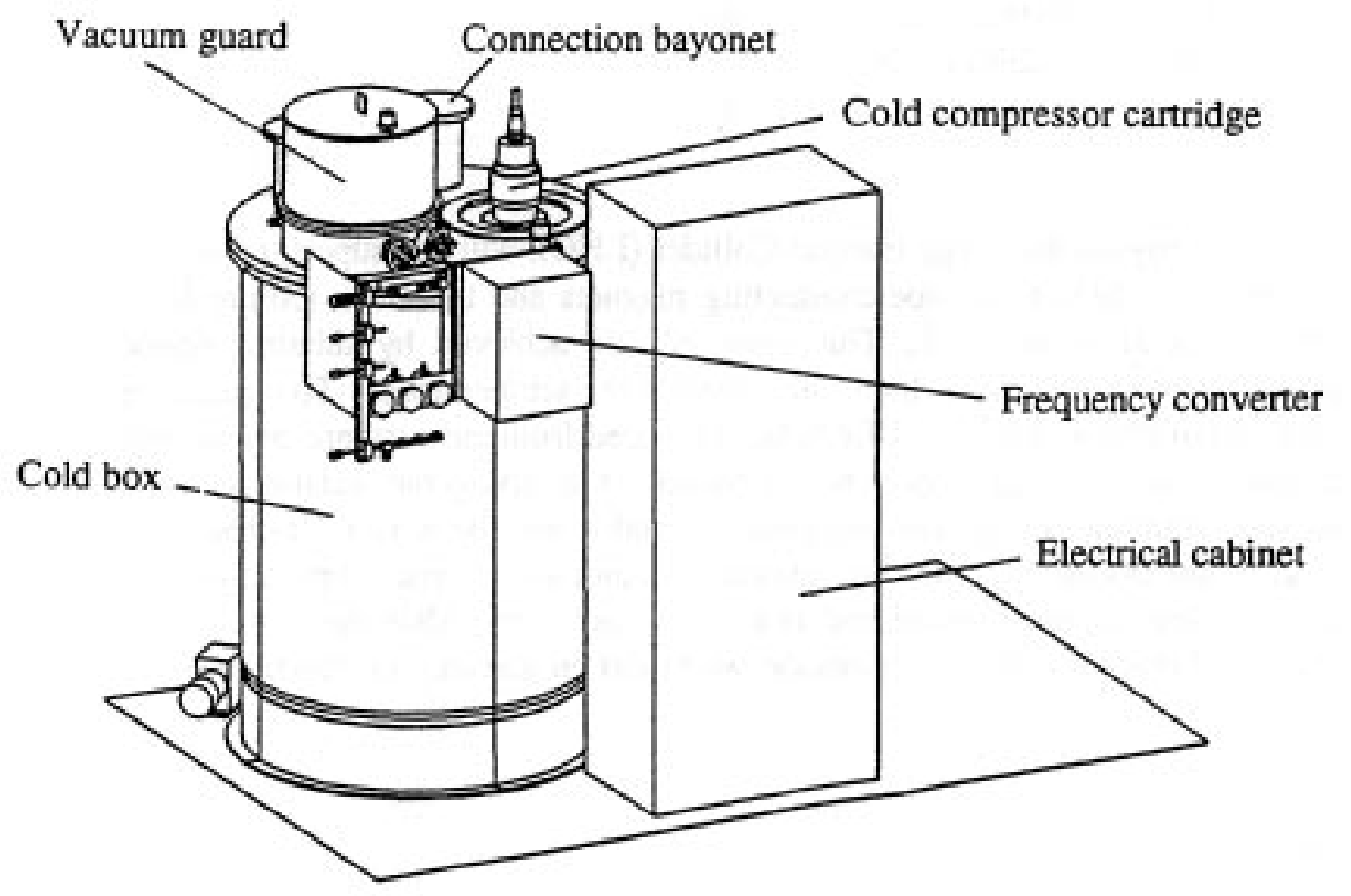

Figure 1 General layout of the $\mathrm{CCU}$

Cold compressor description [3]

The axial-centrifugal unshrouded wheel with a diameter of $118 \mathrm{~mm}$ is fixed to the bearing shaft by a titanium tube giving reduced heat inleaks. Ceramic ball bearings and a variable-frequency electrical motor drive operate under low-pressure helium at ambient temperature. Motor drive, bearings and wheel compose the cartridge. The cold part constituted by the diffuser and volute remains inside the CCU cold box and is supported from the top flange by a thin-walled stainless steel tube.

\section{Instrumentation}

Efficiency assessment of the cold compressor requires accurate temperature and pressure measurements at inlet and outlet. Two calibrated germanium temperature sensors and two absolute pressure gauges have been chosen to fulfill this requirement. Other standard pressure and temperature measurements allow to monitor purge, cooldown, pumpdown and warmup. To avoid risk of air inleaks, all warm components which are working below atmospheric pressure are placed in a vacuum guard.

\section{COMMISSIONING PROGRESS}

Commissioning was performed in two steps. During August 1995, pressure and leak tightness tests as well as piping integrity were checked. Cooldown and warmup were also performed. Initial problems of electrical breakdown in very-low pressure helium in the motor housing precluded performance tests. After improving electrical insulation, a second test campaign took place in November 1995 to complete commissioning. 


\section{RESULTS}

Cooldown and warmup

These two operations take a few hours each. Cooldown is produced by the helium flow pumped by the WPU in series with the CCU. During this phase, the two insulation valves are open, the drive speed is fixed to $100 \mathrm{~Hz}$ and the bypass valve controls the pressure difference between inlet and outlet of the compressor. For warmup, the CCU is completely stopped. A small flow of warm helium gas is injected at the CCU inlet and discharged at the outlet in a recovery line.

$\underline{\text { Static heat inleaks }}$

Heat inleaks were assessed by inlet-outlet temperature difference measurement with the compressor stopped and for different flow rates, yielding a value of $50 \mathrm{~W}$. Due to an increased thickness of the volute support tube during manufacturing (to avoid uncertainties from assembly and unexpected problems during tests), the heat load is $16 \mathrm{~W}$ higher and thus affects the global isentropic efficiency.

Nominal and low-flow operation

In steady-state operation, the compressor controls its suction pressure Pin by adjusting the motor drive speed $\mathrm{N}$. The inlet pressure then stays stable within $10 \mathrm{~Pa}$, better than the required value of $50 \mathrm{~Pa}$. For a given mass-flow $\dot{\mathrm{m}}$, the volumetric characteristic of the WPU fixes the CCU outlet pressure and consequently the pressure ratio of the cold compressor. Several measurements were done varying mass flow from 6 to $18 \mathrm{~g} / \mathrm{s}$ and inlet temperature Tin from $3.5 \mathrm{~K}$ to $5.3 \mathrm{~K}$. Table 2 summarized the main results and figure 2 shows the measured points on the calculated compression field. The operating conditions of turbomachines are expressed in terms of reduced mass-flow $\dot{\mathrm{mr}}$ and speed $\mathrm{Nr}$, defined as follows:

$$
\dot{\mathrm{mr}}=\dot{\mathrm{m}} \cdot \sqrt{\frac{\operatorname{Tin}_{\operatorname{Tin}_{0}}}{\operatorname{Pin}_{0}}} \frac{\operatorname{Pin}_{\operatorname{Pr}}}{\mathrm{P}} \quad \text { and } \cdot \sqrt{\frac{\operatorname{Tin}_{0}}{\operatorname{Tin}}} \quad \text { with subscript } 0 \text { for design conditions }
$$

Table 2 Nominal and low-flow measurements

\begin{tabular}{cccccccccc}
\hline Point & $\begin{array}{c}\dot{\mathrm{m}} \\
{[\mathrm{g} / \mathrm{s}]}\end{array}$ & $\begin{array}{c}\text { Tin } \\
{[\mathrm{K}]}\end{array}$ & $\begin{array}{c}\text { Pin } \\
{[\mathrm{kPa}]}\end{array}$ & $\begin{array}{c}\text { Tout } \\
{[\mathrm{K}]}\end{array}$ & $\begin{array}{c}\text { Pressure } \\
\text { ratio }\end{array}$ & $\begin{array}{c}\text { Isentropic } \\
\text { efficiency }\end{array}$ & $\begin{array}{c}\mathrm{N} \\
{[\mathrm{Hz}]}\end{array}$ & $\begin{array}{c}\mathrm{Nr} \\
{[\mathrm{Hz}]}\end{array}$ & $\begin{array}{c}\dot{\mathrm{mr}} \\
{[\mathrm{g} / \mathrm{s}]}\end{array}$ \\
\hline & & & & & & & & & \\
1 & 8.1 & 4.42 & 1 & 6.55 & 1.25 & 0.19 & 228 & 203 & 9.10 \\
2 & 10.2 & 3.85 & 1 & 6.09 & 1.57 & 0.34 & 265 & 252 & 10.70 \\
3 & 12.2 & 4.68 & 1 & 7.74 & 1.92 & 0.46 & 353 & 306 & 14.11 \\
4 & 14.2 & 4.34 & 1 & 7.60 & 2.27 & 0.52 & 376 & 338 & 15.81 \\
5 & 16 & 3.69 & 1 & 6.80 & 2.57 & 0.54 & 367 & 358 & 16.43 \\
6 & 18 & 3.46 & 1 & 6.75 & 2.95 & 0.57 & 381 & 384 & 17.90 \\
7 & 18 & 4.17 & 1 & 8.16 & 2.95 & 0.56 & 430 & 394 & 19.65 \\
8 & 18.2 & 3.31 & 1 & 6.52 & 2.98 & 0.56 & 375 & 385 & 17.70 \\
9 & 18 & 2.87 & 0.95 & 6.03 & 3.40 & 0.57 & 366 & 404 & 17.16 \\
\hline
\end{tabular}

Isentropic efficiency

The measured isentropic efficiency is slightly below specification. The measurement error, which depends on those on temperature and pressure, is estimated to \pm 0.02 . The higher static heat inleaks to the cold compressor, which account for a loss of efficiency between 0.03 and 0.06 , depending upon their exact location, may thus explain the difference to the specified efficiency. Possible corrective actions are being investigated by the manufacturer. At low flow, the relative proportion of the static heat loads in the loss of efficiency increases and below $14 \mathrm{~g} / \mathrm{s}$, it is no longer possible to obtain the specified value of 0.5. 
$\underline{\text { Stall line measurement }}$

By adjusting a bypass valve on the WPU, it was possible to run the cold compressor at constant pressure ratio with smaller mass flow. In this way, one can reach and explore the stall limit of the wheel. Figure 2 shows the good agreement between the calculated and measured stall limits.

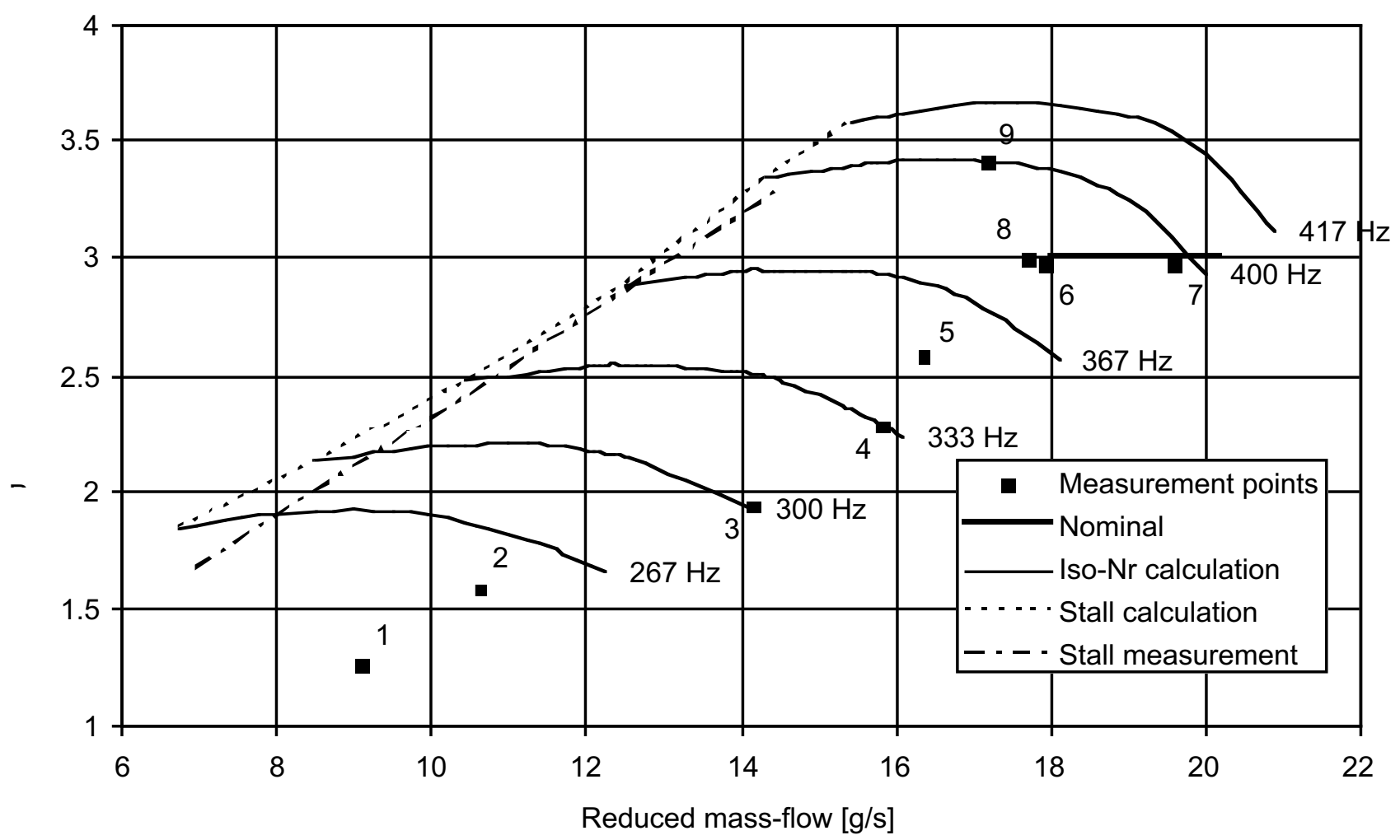

Figure $2 \mathrm{CCU}$ reduced compression field (parameter is reduced speed $\mathrm{Nr}$ ).

Endurance test

In the course of the second test campaign, the CCU was operated in fully automatic mode, for a total of 50 hours without interruption. This test gives confidence in the future use of the CCU but more operating experience is required in order to qualify the long-term reliability of the bearings and motor drive. Identical motor and bearings installed in a test rig simulating operating conditions at Linde's have so far gone through more than 6000 hours of continuous operation without any problem.

\section{CONCLUSION}

With the exception of a slightly lower than specified isentropic efficiency, the CCU supplied by Linde, ATEKO and PBS fulfills the technical requirements. Studies to improve its performance are under way. Moreover, additional tests are in progress to qualify long-term performance and reliability of the drive and bearing system.

\section{ACKNOWLEDGMENTS}

The authors wish to acknowledge contributions of A. Bézaguet, S Claudet, D. Lavielle, and B. Vullierme to the preparation and execution of the cold compressor tests. 


\section{REFERENCES}

1 The LHC Study Group, The Large Hadron Collider, Conceptual Design, CERN Report AC/95-05(LHC) (1995).

2 Benda, V., Dauvergne, J.P., Haug, F., Knoops, S., Lebrun, Ph., Sergo, V., Tavian, L., and Vullierme, B., Upgrade Of The CERN Cryogenic Station for Superfluid Helium Testing of Prototype LHC Superconducting Magnets, paper presented at this conference.

3 Schustr, P., Vin_, M., Brunovsky, I. and Tucek, L., Helium Low Temperature compressor, Proc. Kryogenika 96, Prague (1996) 